\title{
Methicillin Resistant Staphylococcus aureus: Prevalence and Antibiogram in Various Clinical Specimens at Alka Hospital
}

\author{
Kushal Shahi ${ }^{1}$, Komal Raj Rijal ${ }^{1 *}$, Nabaraj Adhikari ${ }^{1}$, Upendra Thapa Shrestha ${ }^{1}$ Megha Raj \\ Banjara $^{1}$, Vijay K Sharma ${ }^{2}$, Prakash Ghimire ${ }^{1}$ \\ ${ }^{1}$ Central Department of Microbiology, Tribhuvan University, Kirtipur, Nepal \\ ${ }^{2}$ Institute of Medicine, Tribhuvan University, Maharajgunj, Kathmandu, Nepal
}

*Corresponding author: Dr. Komal Raj Rijal, Lecturer, Central Department of Microbiology, Tribhuvan University, Kirtipur, Kathmandu, Nepal; E-mail: rijalkomal@yahoo.com

\begin{abstract}
Objectives: This study aimed to study the prevalence and antibiotic susceptibility pattern of methicillin-resistant Staphylococcus aureus (MRSA) isolated from clinical specimen.

Methods: During the study period (April-September, 2013), 754 various clinical samples collected from patients visiting at Alka Hospital were cultured for isolation of S. aureus. The isolates were characterized as S. aureus by their morphology on Gram staining, growth characteristics and coagulase production. Screening of methicillinresistant $S$. aureus was determined using cefoxitin disk as recommended by Clinical Laboratory Standard Institute. All isolates were subjected to antimicrobial susceptibility testing by modified Kirby Bauer disc diffusion methods.

Results: Total 109 (14.4\%) isolates were confirmed as S. aureus and 36 (33.0\%) S. aureus isolates of them were screened as methicillin resistant S. aureus. Maximum percentage $(63.9 \%)$ of methicillin resistant S. aureus were comprised of pus specimens. Highest percentage (47.6\%) of MRSA was isolated from the age group of above 60 years. Maximum percentage of MRSA (63.9\%) was detected in admitted patients. Majority of MRSA isolates were observed to be multidrug resistant. All 36 isolates of MRSA were sensitive to vancomycin. Beside vancomycin, ceftriaxone $(83.3 \%)$ found to be most effective drug for the MRSA isolates.
\end{abstract}

Conclusion: The emergence of drug resistance and its dissemination in MRSA is worrisome. So we need to develop newer agents as well as slow down the spread of resistant strains by various control measures.

Key words: Antibiogram, S. aureus, MRSA.

\section{INTRODUCTION}

Antibiotic resistance is a serious and growing phenomenon in contemporary medicine and has emerged as one of the eminent public health concerns of the $21^{\text {st }}$ century as result of antibiotic pressure. During the past four decades, Methicillin-Resistant S. aureus (MRSA) has evolved from a controllable nuisance into a serious public health concern (Singh et al. 2014). MRSA is one of the potent pathogens causing a variety of infections ranging from relatively benign skin diseases to life-threatening infections such as

Date of Submission: November 12, 2018

Published Online: January 2019 pneumonia, meningitis, endocarditis and septicemia (Baorto 2014). MRSA was first reported in 1961in United Kingdom, just one year after launch of methicillin and outbreaks of MRSA infections were reported in Europe soon thereafter (Davies and Davies 2010). Later it has been emerged as one of leading nosocomial pathogens for past 50 years and emerged in the community as well (Gopalakrishnan and Sureshkumar 2010). MRSA accounts more than $50 \%$ of nosocomial infection (Venkatesh et al. 2014). MRSA infections are a global problem across health economy as it has been associated with significant morbidity, mortality, a poorer outcome

Date of Acceptance: December 8, 2018

DOI: https://doi.org/10.3126/tujm.v5i0.22316 
and higher costs (Soltani et al. 2010). Fighting MRSA involves re-enforcement of infection control measures as well as rational use of antimicrobials. Communityassociated cases of MRSA (CA-MRSA) were reported starting in the late 1990s (DeLeo et al. 2010). CA-MRSA infections were first described in pediatric patients only but now have become a significant public health threat. CA-MRSA posses Mec (SCCmec) types IV and V, that are resistant to fewer antimicrobial agents and exhibit enhanced virulence. CA-MRSA infections typically occur as skin or soft tissue infections but can develop into more invasive and serious infections. CA-MRSA tends to occur more in conditions where people are in close physical contact such as long-term care facilities (Raygada and Levine 2009).

In MRSA, the horizontally acquired $M e c A$ gene encodes a penicillin-binding protein ( $\mathrm{PBP} 2 \mathrm{a})$, which has low affinity to all $\beta$-lactams, responsible for resistance. The MecA gene complex also contains insertion sites for plasmids and transposons that facilitate the acquisition of resistance to other antibiotics (Dominguez 1997). Resistance to multiple antibiotics among MRSA isolates is very common and has posed a serious therapeutic challenge becoming a problem of global extent. MRSA is of serious concern because of resistance to many antimicrobials that are used on a regular basis in hospitals limiting therapeutic options and increasing cost of treatment. Presently MRSA isolates have been uniformly susceptible only to glycopeptides, considered as last resort for these strains. Clinical isolates of MRSA with reduced susceptibility to glycopeptides were first described in Japan in 1997 (Hiramatsu et al. 1997). Vancomycin resistant S. aureus is not widely seen even though a low-level resistance to vancomycin is being reported. The prolonged hospitals stay and indiscriminate are the possible predisposing factors of MRSA emergence. Asymptomatically colonized healthcare workers are the major sources of MRSA in the hospital environment (Orji et al. 2012).

MRSA are prevalent throughout the world. In US about $40 \%$ of $S$. aureus infections acquired are found to be methicillin-resistant (Zaoutis TE 2013). The percentage of hospitals isolating MRSA in the developed countries has increased from $2 \%$ in the $70^{\prime}$ s to $30 \%$ in the 90 's (Gordon 1993). MRSA is now endemic in Nepal. The growing problem in the Nepalese scenario is that
MRSA prevalence is rapidly increasing with the time. The incidence of MRSA in Nepal varied from $11.76 \%$ in 1999 reported by Lamichhane to 60\% in 2010 observed by Khanal and Jha carried out in a tertiary-care hospital in Eastern Nepal. These studies clearly show the need of efficacious and rapid infection control measures. The present study provides a hospital level initiative to understand emerging trends of antimicrobial resistance among local MRSA isolates and provides a platform to initiate epidemiological studies for MRSA infections. The current status of antimicrobial susceptibility profile of local MRSA isolates is essential for the selection of appropriate therapy for the management of infections. Data from such study can be utilized to formulate cost effective empirical therapy and make better hospital infection control policies. A hospital level study helps to know the best treatment options available for MRSA infected patients.

\section{MATERIALS AND METHODS}

\section{Study population}

This was a prospective descriptive study carried out at Alka hospital, Lalitpur. Participants were the patients visiting Alka hospital. All clinical specimens were obtained from participants for study.

\section{Conventional microbiological tests}

All clinical specimens collected aseptically were processed and cultured using standard microbiological procedures. Isolated colonies from the pure culture were identified by performing the standard conventional biochemical tests. Susceptibility tests of the different clinical isolates towards various antibiotics were performed by modified Kirby-Bauer M02-A9 disk diffusion method using Mueller Hinton Agar (MHA). MRSA isolates in pure culture were preserved in $20 \%$ glycerol containing tryptic soya broth and kept at $-70^{\circ} \mathrm{C}$ until subsequent tests were performed (CLSI 2007).

Detection of methicillin resistant Staphylococcus aureus isolates

The methicillin resistant Staphylococcus aureus isolates were screened using cefoxitin disk $(30 \mu \mathrm{g})$. The screened isolates were subjected to antimicrobial susceptibility testing by modified Kirby Bauer disc diffusion methods for the determination of current antimicrobial susceptibility pattern of local MRSA isolates (CLSI 2007). 


\section{RESULTS}

MRSA profile in different specimens

Out of total 754 various clinical samples processed and cultured for isolation of Staphylococcus aureus, 109

$(14.4 \%)$ isolates were confirmed as S. aureus. Out of them $36(33.0 \%)$ isolates were screened as methicillin resistant Staphylococcus aureus. Maximum percentage $(63.9 \%)$ of methicillin resistant S. aureus strains were comprised of pus specimens (Table 1).

Table 1: Distribution of MRSA in different specimens

\begin{tabular}{lccc}
\hline Specimens & S. aureus, $\mathrm{n}(\%)$ & MRSA & MSSA \\
\hline Pus & $77(70.6)$ & $23(63.9)$ & $54(73.9)$ \\
Urine & $13(11.9)$ & $6(16.7)$ & $7(9.6)$ \\
Sputum & $8(7.3)$ & $5(13.9)$ & $3(4.1)$ \\
Ear swab & $4(3.7)$ & 0 & $4(5.5)$ \\
Plural fluid & $3(2.8)$ & $1(2.8)$ & $2(2.8)$ \\
Other & $4(3.7)$ & $1(2.8)$ & $3(4.1)$ \\
\hline Total & $109(100)$ & $36(33.03)$ & $73(66.97)$ \\
\hline
\end{tabular}

Distribution of MRSA isolates among outdoor and indoor patients in different age groups

Out of total 36 MRSA strains isolated, 14 (38.9\%) strains were isolated from outpatients whereas 22 (61.1\%) strains of MRSA were isolated from admitted patients. The highest percentage of MRSA (27.8\%) was observed in the age group of above 60 years (Table 2).

Table 2: MRSA among outdoor and indoor patients in different age groups

\begin{tabular}{lcccc}
\hline \multirow{2}{*}{ Age (Years) } & \multicolumn{2}{c}{ Outpatients } & \multicolumn{2}{c}{ Indoor patients } \\
\cline { 2 - 5 } & S. aureus n(\%) & MRSA & S. aureus & MRSA \\
\hline$<10$ & $1(2.2 \%)$ & 0 & $10(15.9 \%)$ & $4(18.2 \%)$ \\
$11-20$ & $6(13 \%)$ & $4(28.5 \%)$ & $7(11.2 \%)$ & $1(4.6 \%)$ \\
$21-30$ & $11(23.9 \%)$ & $1(7.1 \%)$ & $13(20.6 \%)$ & $3(13.6 \%)$ \\
$31-40$ & $2(4.3 \%)$ & $1(7.1 \%)$ & $5(7.9 \%)$ & $1(4.6 \%)$ \\
$41-50$ & $5(10.9 \%)$ & $2(14.3 \%)$ & $13(20.6 \%)$ & $5(22.7 \%)$ \\
$51-60$ & $10(21.8 \%)$ & $2(14.3 \%)$ & $5(7.9 \%)$ & $2(9 \%)$ \\
$>60$ & $11(23.9 \%)$ & $4(28.7 \%)$ & $10(15.9 \%)$ & $6(27.3 \%)$ \\
\hline Total & $46(42.2 \%)$ & $14(38.9 \%)$ & $63(57.8 \%)$ & $22(61.1 \%)$ \\
\hline
\end{tabular}

Antibiotic susceptibility pattern of isolates

All 36 strains of MRSA were sensitive to vancomycin. Beside vancomycin, ceftriaxone found to be most effective for the MRSA strains (75\%), followed by levofloxacin
(69.4\%), tetracycline (66.7\%), amikacin $(66.7 \%)$ and ciprofloxacin (63.9\%). Similarly, most resistant drug among the MRSA strains was amoxycillin (91.6\%) and cloxacillin (88.8\%) (Table 3)

Table 3: Antibiotics resistance pattern of MRSA isolates

\begin{tabular}{lcc}
\hline Antibiotics & S.aureus, $\mathrm{n}(\%)$ & MRSA, $\mathrm{n}(\%)$ \\
\hline Amoxycillin & $62(56.9)$ & $33(91.6)$ \\
Ciprofloxacin & $35(32.1)$ & $13(36.1)$ \\
Erythromycin & $39(35.8)$ & $17(47.2)$ \\
Levofloxacin & $52(22.9)$ & $11(30.6)$ \\
Cloxacillin & $50(45.9)$ & $32(88.8)$ \\
Cefoxitin & $36(33)$ & $36(100)$ \\
Amikacin & $34(31.2)$ & $12(33.3)$ \\
Vancomycin & 0 & 0 \\
Ceftriaxone & $24(22)$ & $9(25)$ \\
Chloramphenicol & $31(28.4)$ & $15(41.6)$ \\
Tetracycline & $27(24.8)$ & $12(33.3)$ \\
\hline
\end{tabular}




\section{DISCUSSION}

MRSA has emerged as a serious threat to public health worldwide. It has added to the burden of patient by prolonging hospital stay and increasing morbidity, mortality rate and cost. Present study showed prevalence rate of MRSA to be $33.03 \%$. This finding is consonent with the finding of Fayomi et al. (2009), in this study which is carried out in Ido-Ekiti, Nigeria; they found $31 \%$ MRSA. The result obtained is in agreement with the findings of Vidya et al. (2010), who reported 29.1\% MRSA isolates in Mangalore, South India. Likewise, our finding is similar with the finding of Mir (2013), he reported the prevalence of MRSA to be $32.2 \%$ in Pesawar, Pakistan. In Nepal, these findings also synchronize with the findings of Sapkota (2006), who reported 31.1\% MRSA and Thapa (2004) reported $29.23 \%$ MRSA. On the contrary, report shown by Rajbhandari (2002), has alarmingly high incidence of MRSA infection (54.9\%). Our outcome is belied with the findings of Rijal et al. (2008), who reported 75.5\% MRSA in a study conducted in Pokhara Valley. Similar study done in western parts of Nepal by Tiwari et al. also had shown alarmingly high rate of MRSA isolate (69.1\%) in 2009 which the authors has attributed to indiscriminate use of antibiotics and its accessibility in these areas (Tiwari et al. 2009). Above studies show considerable variations between institutions, often in the same geographical areas, demonstrating that MRSA prevalence, in some settings, significantly exceeds previous estimate. There could be many explanations for these differences: infection control measures, antibiotic prophylaxis and treatments used in each hospital and, not less important, the clonal and often epidemic nature of these microorganisms (Rijal et al. 2008). This study showed maximum percentage of $S$ aureus $(70.6 \%)$ and MRSA (63.9\%) isolated from pus ascertaining the role of the organism as cause of pyogenic infection. This is similar to the study done in Nepal, India and Pakistan (Shrestha et al. 2009). This study observed highest percentage $(47.6 \%)$ of MRSA was isolated from the age group of above 60 years. This might be due to the high dose of medication because of difficulties in infection treatment in that group. Furthermore, the higher vulnerability of such age group can be correlated with the reduced immune system in that group of patients.
This result is consonant with finding of Duran et al. (2012). Higher percentage of MRSA was isolated from admitted patients that suggest high antibiotic pressure in hospital and hence they are more prone to be MRSA. This is harmony with report of Adeleke and Olarinde (2013). However in remote regions of Nepal where availability and use of antibiotics is limited, the prevalence of MRSA was observed to be low, reported by Subedi and Brahmadath in 2005.

Analysis from previous studies revealed a relationship between methicillin resistance and resistance to other antibiotics. This study showed that all MRSA isolates were significantly less sensitive to antibiotics. Homogeneous insusceptibility to beta-lactams like amoxycillin and cloxacillin, resistant MRSA was also observed in our study. This may be due to presence of intrinsically developed beta-lactamase in MRSA strain. It also showed the high resistance to erythromycin as such antibiotics are usually used at random to cure generalized and pyogenic infection. Antimicrobials such as amikacin and tetracycline with resistance less than $35 \%$ could be used against of MRSA infection. But due to their mode of action, have limited use for empirical therapy of MRSA related infection. Resistance to ceftriaxone was observed to be $25 \%$ in this study. Limiting its indiscriminate use and doing antibiotic susceptibility testing, it could be considered for empirical therapy for MRSA infection in this setting. The multi-drug resistant has become in methicillinresistant S.aureus strains. It has added burden to hospital personal in infection control and has limited therapeutic option. In this study, majority of MRSA isolates were MDR. Studies conducted in eastern and western part of Nepal also reported MDR-MRSA to be as high as $65-78 \%$. Though these MDR strains are not found with additional virulence properties, multidrug resistance only restricts the options available to treat infections caused by this organism (Kumari et al. 2008). Vancomycin, a glycopeptide seems to be the only antimicrobial agent which showed $100 \%$ effectiveness through all parts of Nepal and may be used as the drug of choice for treating multidrug resistant MRSA infections, it should be preserved for life threatening infection. But its toxic side effects like renal impairment and high cost has limited its use. When vancomycin is 
considered for treatment, in vitro susceptibility testing is most owing to emergence of Vancomycin resistant Staphylococcus aureus (VRSA) in various parts of world (Sakoulas and Moellering 2008).

\section{CONCLUSION}

Existence of MRSA isolates is a serious matter of concern. Moreover, drug resistance in MRSA at study area is worrisome in the current therapeutic scenario as majority of MRSA isolates were multidrug resistant. These findings call for urgent attention on regular surveillance in antibiotic profile of Staphylococcus isolates. Vancomycin still remains the drug of choice for MRSA infection; it should be preserved for lifethreatening MRSA infection. Ceftriaxone promises to be the best for the treatment of MRSA isolates in the study area in vitro.

\section{CONFLICT OF INTEREST}

The authors don't have any conflict of interest.

\section{REFERENCES}

Baorto EP (2014). Staphylococcus aureus infection. Medscape 2: 50-59.

Clinical Laboratory Standard Institute (2007). Performance standards for antimicrobial susceptibility testing; Seventeenth informational supplement-Clinical Laboratory Standard Institute M100-S17 27: 1.

Davies J and Davies D (2010). Origin and evolutions of antimicrobial resistance. Microbiol Mol Biol Rev 74(3): 417-433.

DeLeo FR, Otto M, Kreiswirth BN and Chambers HF (2010). Community-associated methicillin resistant Staphylococcus aureus. Lancet 375: 15571568.

Domínguez MA, Liñares J and Martín R (1997). Molecular mechanisms of methicillin resistance in Staphylococcus aureus. Microbiologia 13(3): 301308.

Gopalakrishnan R and Sureshkumar D (2010). Changing trends in antimicrobial susceptibility and hospital acquired infections over an 8-year period in a tertiary care hospital in relation to introduction of an infection control programme.
J Assoc Physicians India 58 (Suppl): 25-31.

Gordon J (1993). Clinical significance of methicillin sensitive and methicillin resistant Staphylococcus in UK Hospitals and the relevance of povidone iodine in their control. Postgrad Med J 69 (3): 106116.

Hiramatsu K, Aritaka N, Hanaki H, Kawasaki S, Hosoda Y, Hori S, Fukuchi Y and Kobayashi I (1997). Dissemination in Japanese hospitals of strains of Staphylococcus aureus heterogeneously resistant to vancomycin. Lancet 350: 1670-1673.

Kumari N, Mohapatra TM and Singh YI (2008). Prevalence of Methicillin-Resistant Staphylococcus aureus (MRSA) in a Tertiary-Care Hospital in Eastern Nepal. J Nepal Med Assoc 47: 53-56.

Orji I, Nworie A, Eze UA, Agberotimi IO, Okereke EC and Azi SO (2012). The prevalence and antimicrobial susceptibility profile of methicillin resistant Staphylococcus aureus isolates from clinical specimens in a tertiary hospital, south east Nigeria. Continental J Pharm Sci 6(1), 23-29.

Raygada JL and Levine DP (2009). Managing CAMRSA Infections: Current and emerging options. Inf Med 26: 42-44.

Rijal KR, Shrestha N, Pahari N, Shrestha B, Paudel B, Nepal A, Ghimire P and Rijal B (2008). Methicillin resistant Staphylococcus aureus in patients visiting Western region hospital. JIOM 30(1): 21-25.

Sakoulas G and Moellering RC Jr (2008). Increasing antibiotic resistance among methicillin-resistant Staphylococcus aureus strains. Clin Infect Dis 46(5): 360-367.

Shrestha B, Pokhrel BM and Mohapatra TM (2009). Phenotypic Characterization of nosocomial isolates of Staphylococccus aureus with reference to MRSA. J Infect Dev Ctries 3(7): 554-560.

Singh H, Atray M and Modi PK (2014). Antimicrobial susceptibility pattern of methicillin resistant Staphylococcus aureus (MRSA) isolates in tertiary care center at southern Rajasthan. Int J Pharm Sci Res 5(2): 607-611. 
Soltani R, Khalilia H, Rasoolinejadb M, Alireza AbdollahicAandGholamiaK(2010). Antimicrobial susceptibility pattern of Staphylococcus aureus strains isolated from hospitalized patients in Tehran, Iran. Iranian J Pharm Sci 6(2): 125-132.

Tiwari HK, Das AK, Sapkota D, Sivrajan KK and Pahwa VK (2009). Methicillin resistant Staphylococcus aureus: prevalence and antibiogram in a tertiary care hospital in western Nepal. J Infect Dev Ctries 3(9): 681-4
Venkatesh VN, Shwetha DC, Ravish KM, Sudhindra KS and Srinivasa S (2014). MRSA study of antimicrobial susceptibility pattern of methicillinresistant Staphylococcus aureus at tertiary care hospital in south India. J Biomed Pharma Res 3: 25-28.

Zaoutis TE (2013). Rates of serious MRSA infections in children increased about 10\% per year since 2005 . Am Acad Pedria 34: 22-28. 